\title{
Uso de Paquetes Informáticos Para el Análisis de Datos en Ciencias Biomédicas y Sociales
}

\section{Use Packages Software For The Analysis Of Data In Biomedical Sciences And Social}

Díaz Pérez $\mathrm{A}^{1}$, Oñate Díaz $\mathrm{G}^{2}$, Riquena Robles $\mathrm{N}^{2}{ }^{2}$

${ }^{1}$ Universidad Simón Bolívar (Col), ${ }^{2}$ Universidad de la Guajira (Col)

Resumen - La utilización de paquetes informáticos para el análisis de datos cualitativos como cuantitativos en los últimos años ha tornado una gran relevancia al momento de decidir el tipo de publicación 0 tener un mayor impacto al momento de la socialización. Sin embargo los programas no deben remplazar en ningún momento la capacidad de análisis y reflexión de los investigadores, ni ser excusas para un mal diseño metodológico, donde una falta de entrenamiento en el manejo de los paquetes puede llegar a confundir al investigador en relación a la interpretación de los resultados finales.

Palabras Clave: Análisis cualitativo, análisis cuantitativo, registros, apoyo a la investigación.

Abstract - The uses of software a package for the analysis of qualitative data, quantitative in recent years has become a highly relevant at the time of deciding the type of publication or have a major impact at the moment of socialization. However programs should not replace the capacity for analysis and reflection of the researchers at any time or be excuses for a poor methodological design, where a lack of training in the management of packages can mislead the researcher in relation to the interpretation of the final results.

Keywords: Qualitative analysis, quantitative analysis, records, research support.

\section{INTRODUCCIÓN}

El modo de diseñar y analizar los resultados de cualquier investigación científica ha cambiado considerablemente en el último siglo, los avances en el campo de la metodología, el desarrollo de software estadístico con el manejo de la informática es determinante para la publicación y socialización en eventos científicos $(1,2)$.

Los procedimientos que actualmente se presentan con mayor frecuencia en la literatura biomédica son modelos de regresión logística "regresión de riesgos proporcionales" o herramientas que permiten el análisis de datos procedentes de revisiones altamente complejas y dinámicas como son los estudios de supervivencia, metadatos y experimentales. A nivel mundial se exige que las publicaciones de los artículos originales ya sean

Correspondencia: Anderson Díaz Pérez. Programa de instrumentación quirúrgica. Universidad Simón Bolívar. Carrera 59 No. 59-65 Barranquilla, Atlántico, Colombia. ander2711@gmail.com.

Citar: Díaz A, Oñate G, Riquena N. Uso de paquetes informáticos para el análisis de datos en ciencias biomédicas y sociales. Cienc. innov. salud. 2015; 3 (2):43 - 49.

Recibido: Feb. 01 de 2015 / Modificado: Jun. 01 de 2015 / Aceptado: Jul. 21 de 2015. de naturaleza cuantitativa o cualitativa se hayan procesado y analizados a partir de software original, altamente eficiente, potente, confiable y validado a través de investigaciones científicas en donde se analicen sus características y funciones, como por el ejemplo el SPSS en lo cuantitativo y en lo cualitativo se encuentre el NUD*IST VIVO el ATLAS Ti, WinMAX, AQUAD, ETHNOGRAPH, MAXQDA, entre otros.

\section{Tipos de Modelos estadísticos y temas de FoRM ACIÓN CIENTÍFICA}

Los artículos publicados deben contener ciertos cálculos estadísticos altamente sofisticados que permiten inferir eficientemente la muestra, comparar poblaciones en relación a tratamientos e intervenciones, en cualquier de los métodos de estudios sean estos "epidemiológicos o ensayos clínicos". Las revistas exigen un análisis de acuerdo al nivel de reflexión de los resultados, sugiriendo algunos análisis estadísticos de acuerdo incluso al tipo de indexación de la revista a la cual se proyecta la publicación o socialización de los productos resultados e investigación (2-4). (Tabla 1. Protocolo para el análisis estadístico en artículos de investigación original).

El filtraje de los manuscritos mediante revisión específica por expertos (pares evaluadores), empezando por el mismo editor el cual requiere entrenamiento especial en análisis estadísticos, filtran la publicación de artículos, poster o ponencias para participar en eventos y publicaciones nacionales e internacionales; donde un mal análisis estadístico o una interpretación errónea a los cálculos es un factor suficiente para el rechazo de los artículos, así como por falencias complementarias, en lo que respecta a la metodología: hipótesis no contrastadas, protocolo original modificado, control de sesgos inadecuado, diseño y/o análisis estadísticos incorrectos 0 inapropiados, tamaño muestral insuficiente 0 conclusiones liberales al margen de los resultados observados. 
Tabla 1. Protocolo diseñado por Emerson y Colditz y adaptado por Mora I Ripoll y cols. Niveles de referencia para el análisis de accesibilidad

\begin{tabular}{|c|c|c|}
\hline CRITERIO & DESCRIPCIÓN & ACLARACIONES \\
\hline 1 & $\begin{array}{l}\text { Estadística } \\
\text { Descriptiva }\end{array}$ & $\begin{array}{l}\text { Ningún contenido } \quad \text { estadístico } \\
\text { únicamente estadística descriptiva }\end{array}$ \\
\hline 2 & $\begin{array}{l}\text { Prueba } t \text { de Student } t \\
\text { pruebas } z\end{array}$ & $\begin{array}{l}\text { Para una muestra o dos muestras (datos } \\
\text { apareados y/ o independientes) }\end{array}$ \\
\hline 3 & Tablas bivariables & $\begin{array}{l}\text { JI cuadrado, prueba exacta de Fisher, test } \\
\text { de } \mathrm{M} \text { cNemar }\end{array}$ \\
\hline 4 & Test no paramétricos & $\begin{array}{l}\text { Test de los signos, U de Mann-Whitney } \\
\text { prueba T de Wilcoxon }\end{array}$ \\
\hline 5 & $\begin{array}{l}\text { Estadísticos } \\
\text { demográficos } \\
\text { epidemiológicos }\end{array}$ & $\begin{array}{l}\text { Riesgo relativo Odds-ratio, Log-Odds, } \\
\text { Medidas de Asociación, Sensibilidad y } \\
\text { Especificidad }\end{array}$ \\
\hline 6 & $\begin{array}{l}\text { Correlación lineal } \\
\text { Pearson }\end{array}$ & $\begin{array}{l}\begin{array}{l}\text { Correlación Clásica (Coeficiente } \mathrm{R} \text { de } \\
\text { correlación lineal) }\end{array} \\
\end{array}$ \\
\hline 7 & Regresión simple & $\begin{array}{l}\text { Regresión de Mínimos cuadrados con una } \\
\text { variable predictora y una respuesta }\end{array}$ \\
\hline 8 & Análisis de varianza & ANOVA, análisis de covarianza pruebas $\mathrm{F}$ \\
\hline 9 & $\begin{array}{l}\text { Transformación de } \\
\text { variables }\end{array}$ & $\begin{array}{ll}\text { Empleos de } & \text { transformaciones } \\
\text { (logarítmicas) } & \end{array}$ \\
\hline 10 & $\begin{array}{l}\text { Correlación } \\
\text { paramétrica }\end{array}$ & $\begin{array}{l}\text { RHO de Spearman. Tau de Kendal Pruebas } \\
\text { de tendencias }\end{array}$ \\
\hline 11 & Regresión M últiples & $\begin{array}{l}\text { Incluye la regresión polinómica y la } \\
\text { regresión pasoa paso }\end{array}$ \\
\hline 12 & $\begin{array}{l}\text { Comparaciones } \\
\text { Múltiples }\end{array}$ & $\begin{array}{l}\text { Gestión de indiferencias múltiples para los } \\
\text { mismos datos; pruebas de Bonferroni, } \\
\text { Scheffer, Duncan, Newman-Keuls. }\end{array}$ \\
\hline 13 & $\begin{array}{l}\text { Ajuste } \\
\text { estandarización }\end{array}$ & $\begin{array}{l}\text { Estandarización de casos de incidencias y } \\
\text { prevalencia }\end{array}$ \\
\hline 14 & Tablas multivariables & $\begin{array}{l}\text { Procedimientos de Mantel Haenszel, } \\
\text { modelos log-lineales }\end{array}$ \\
\hline 15 & $\begin{array}{l}\text { Potencia y tamaño } \\
\text { muestral }\end{array}$ & $\begin{array}{l}\text { Determinación del tamaño de la muestra } \\
\text { en base a una diferencia detectable }\end{array}$ \\
\hline 16 & $\begin{array}{l}\text { Análisis de la } \\
\text { supervivencia }\end{array}$ & $\begin{array}{l}\begin{array}{l}\text { Incluye tablas de vida, regresión de } \\
\text { supervivencia y otros análisis de } \\
\text { supervivencia. }\end{array} \\
\end{array}$ \\
\hline 17 & $\begin{array}{l}\text { Análisis } \\
\text { beneficio }\end{array}$ & $\begin{array}{l}\text { Estimación de los costos de salud para } \\
\text { comparar directrices alternativas (costo- } \\
\text { efectividad) }\end{array}$ \\
\hline 18 & Otros análisis & $\begin{array}{l}\text { Test no incluidos en las categorías } \\
\text { precedentes; análisis de sensibilidad, } \\
\text { análisis de clúster, análisis discriminante... }\end{array}$ \\
\hline & Niveles de Referencia & $\begin{array}{l}\text { I. Hasta la categoría } 0 \text { criterio } 1 \\
\text { II. Hasta la categoría } 7 \text { incluida } \\
\text { (alguna técnica inferencial) } \\
\text { II. Hasta la última categoría (análisis } \\
\text { complejos y multivariables). }\end{array}$ \\
\hline
\end{tabular}

Nivel I: conocimientos sólo hasta la primera categoría ("sólo estadística descriptiva). Nivel II: hasta la categoría 7, correspondiente a un curso básico de estadística (regresión simple). Nivel III: resto de catego rías. Fuente: Autores.

En consecuencia, los autores de artículos presentados a revistas biomédicas deben de prestar un especial énfasis en la perfección de su metodología estadística (diseño y análisis) y tener presente que lo más importante es el diseño del estudio de investigación, ya que un estudio bien diseñado pero pobremente analizado, puede ser siempre rescatado mediante un mejor análisis, mientras que un estudio pobremente diseñado ya no tiene remedio, ni con sofisticadas técnicas estadísticas, por lo que está destinado al fracaso (5). Es decir se requiere una capacitación permanente a los investigadores en el manejo de software o paquetes estadísticos como lo es el SPSS por ser uno de los más utilizados (6).
La investigación biomédica que se diseña, implementa y se publica en las facultades de ciencias de la salud 0 al interior de los grupos de investigación, requiere investigadores capacitados, en los cuales los cursos brindados por las universidades debe ir de la mano de la capacitación independiente por parte de los investigadores con algún tipo de formación en metodología como los coordinadores de investigación, directores de grupos de investigación y personal técnico en investigación, los cuales son al final los directamente responsables de procesar los datos teniendo presente elementos éticos al momento de analizar y divulgar la información. Es por esto que los cursos 0 entrenamientos deben ir enfocados a las principales necesidades del grupo de investigación (6) sin olvidar los principios que regulan la investigación. (Tabla 2 . Principales temas con relación al cálculo estadístico para la formación de investigadores.

Se ha demostrado la necesidad que tienen los investigadores y demás profesionales de la salud dedicados de alguna manera a la actividad investigativa a una formación en temas puntuales asociados con el proceso de investigación (6) (Tabla 3. Temas Sugeridos para desarrollo de los investigadores).

\section{Características y Funciones del Statistical} Package for the Social Sciences (Spss) Spss.

El SPSS se utiliza y permite trabajar en diferentes niveles del proceso investigativo donde se pueden conjugar diferentes modelos estadísticos y cálculos que permiten generalizar los de una muestra a una población más general, así como también en el proceso de tabulación y organización de los datos de acuerdo a la naturaleza y grado de medición de las variables $(3,5-10)$ tales como:
Archivos de datos
Análisis en modo distribuido
Editor de datos
Trabajo con varios orígenes de datos
Preparación de los datos
Transformaciones de los datos
Gestión y transformación de los archivos
Tablas pivote
Modelos estadísticos
Trabajar con sintaxis de comandos
Trabajar con sintaxis de comandos
Puntuación de datos con modelos predictivos
Trabajos de producción
Entre otros 
Tabla 2. Principales temas o secciones de interés para formación en investigación (Gavagnach M. Et al. Formación en investigación: autopercepción de los profesionales sobre sus necesidades) (6).

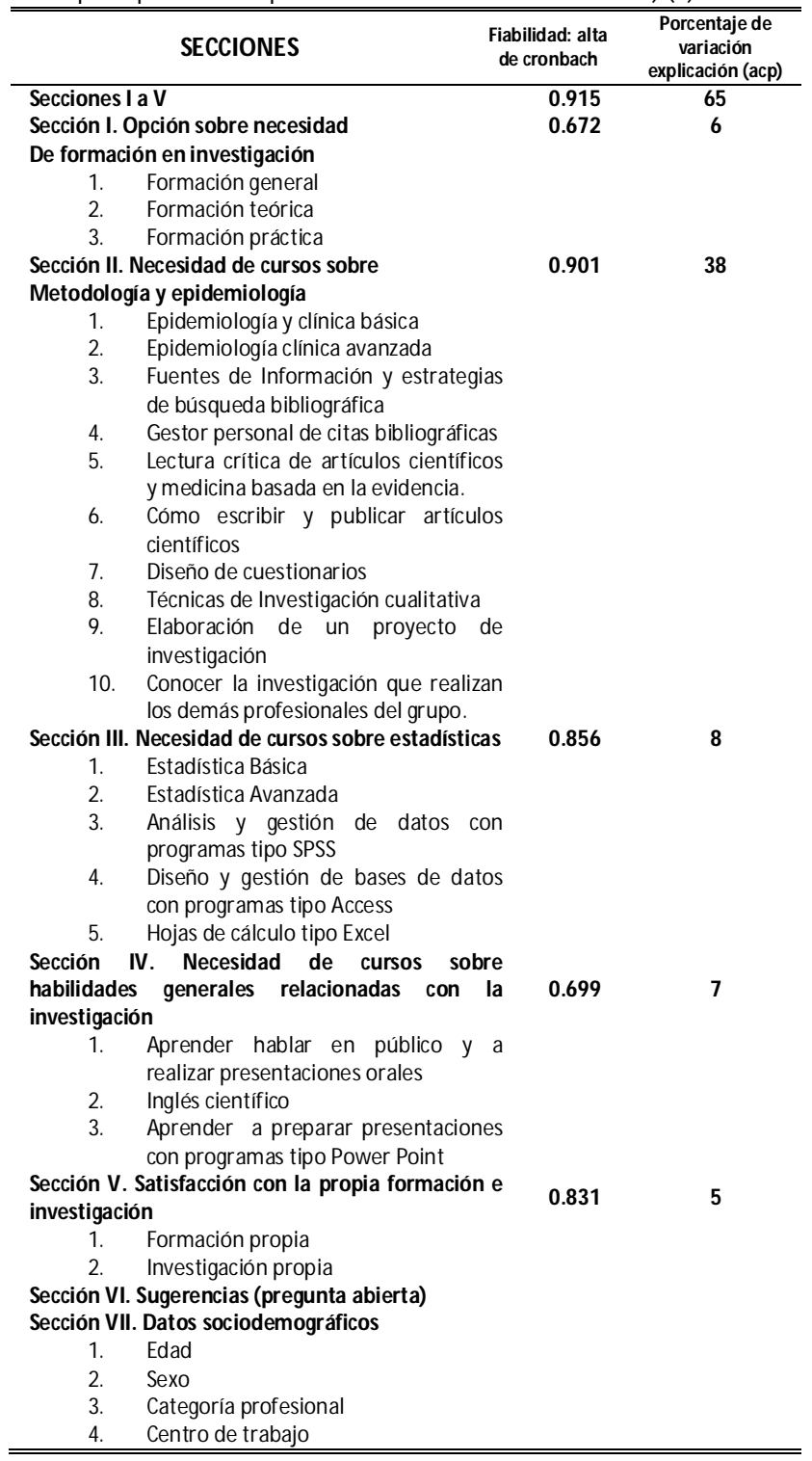

Todas estas características del SPSS permiten procesar y analizar datos en áreas del saber donde se requiera como por ejemplo:

- Lectura de datos

- Transformación de variables

- Recodificación de variables

- Selección de casos

- Estadística descriptiva y gráficos

- Tablas de frecuencias

- Tablas de variables multi-respuesta

- Tablas de doble entrada

- Gráficos

- Histogramas
- Diagrama de barras

- Nubes de puntos

- Gráficos matriciales

- Análisis inferenciales

- Estadística paramétrica

- Estadística no paramétrica

Tabla 3. Temas Sugeridos para desarrollo de los investigadores (6).

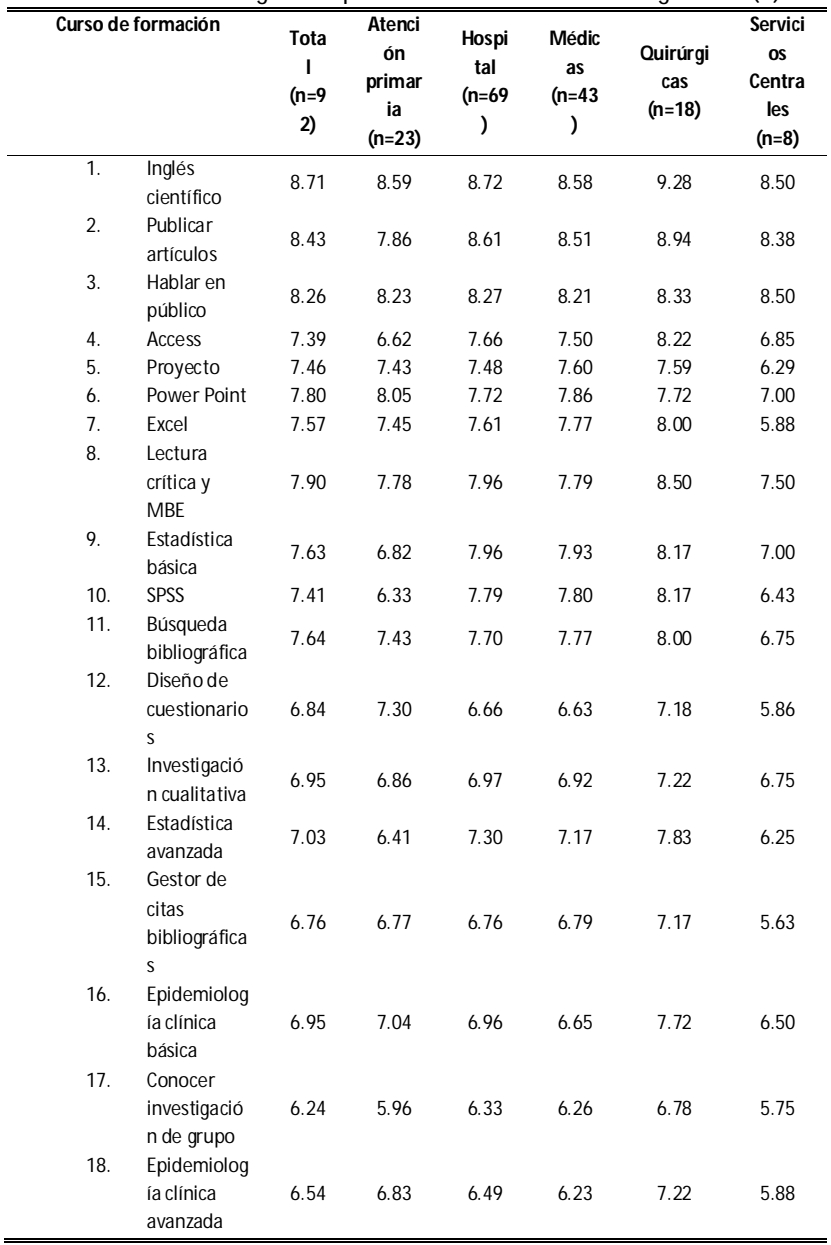

Otros Programas Estadisticos En El Análsis De LA INFORMACIÓN CIENTÍFICA.

En los últimos años se ha venido fomentando el creciente acceso a recursos computarizados, debido al desarrollo de numerosos paquetes estadísticos como él (SPSS, SAS, BMDP, EPINFO, STATGRAPHICS, STATA, etc.) Los cuales cuentan con las pruebas de hipótesis entre sus principales atractivos, el uso de pruebas de significancia, las cuales se han venido casi universalizando en la investigación biomédica. Desde hace varios años diversas revistas de alto rankin en la producción científica internacional dejaron de admitir 
trabajos en los cuales solo aparecían pruebas de tipo descriptivo y sin cálculos complementarios como el intervalo de confianza y no solamente valores de " $p$ " (11).

No hay que olvidar que los análisis estadísticos son solo una herramienta que han de sumarse al arsenal de conocimientos de los científicos e información aportada por estudios anteriores es decir la experiencia, y no configurar una conclusión en información o datos que no pasa de ser un resultado. Es decir; los aplicadores de paquetes estadísticos y verbalizadores de los resultados que dichos paquetes arrojan, necesitan investigadores con gran capacidad analítica que puedan convertir los resultados (estadísticos o no estadísticos) en juicios sustantivos sobre las hipótesis que encaran o las realidades que examinan (11).

\section{LAS TIC EN INVESTIGACIÓN Y USO DE NUD*IST VIVO} Como Herramienta De Perfeccionamiento En INVESTIGACIÓN CUALITATIVA.

El investigador debe estar en capacidad de manejar y analizar una gran variedad de aplicativos, plataformas On Line y paquetes avanzados para el análisis de la información para poder ofrecer a sus estudiantes información actualizada y de impacto académico, así mismo para el diseño de proyectos de investigación, artículos científicos y divulgación en eventos científicos.

El programa NUD*IST Vivo (Nvivo) es uno de muchos programas que se utilizan como apoyo informático en el análisis de datos de naturaleza cualitativa para transcripciones de entrevistas, diarios de campos, registros de observación, etc. $(12,13)$. En muchos universidades del mundo se ha implementado su enseñanza como uno de los contenidos prácticos de las asignaturas de métodos de investigación como contenido del plan de estudios en carreras como la psicopedagogía, psicología, enfermería y medicina, ciencias biológicas, entre otras (12, 14-16).

\section{CARACTERÍSTICAS Y FunCIONES DEL NUd*IST Vivo.}

Desde 1987 basado en el sistema de índice desarrollado por Richards y Richards surge el NU*DIST (No Numerical Unstructured Data, Indexing, Searching and Theorizing). Nudist es un programa previo al surgimiento del NVIVO, ambos producidos por la misma compañía, NVIVO QSR internacional (17).
Siguiendo la estructura explicativa de Valles (2000) se hace una descripción de los principales elementos y funcionalidades del programa NVIVO. A continuación estos aspectos (17):

\section{Creación de documentos compuestos}

En el proyecto se presenta un documento denominado Informe del proyecto, en él se da información sobre el investigador, los objetivos y otros detalles del estudio.

Se trata de un documento textual convencional al que se le han adherido anotaciones.

\section{Mantenimiento de un diario de proyecto}

NVIVO anima a llevar un diario del proyecto, recogiendo así una tradición muy arraigada en el trabajo cualitativo, documento que puede crearse y mantenerse directamente en NVIVO.

Importación de atributos

En general en los estudios cualitativos se dispone de información sobre las personas y los lugares estudiados recabada durante el trabajo de campo. Los atributos de los entrevistados como la edad, sexo, educación, ocupación, etc. y cualquiera atributo que el investigador considere conveniente puede ser incluido en este aspecto. El programa puede crear nuevos atributos y revisar los existentes.

\section{Lectura y codificación de documentos}

NVIVO, sintetiza algunos de los rasgos distintivos más sobresalientes de programas previos, por ejemplo del Atlas ti, recupera el recurso de la codificación in vivo y del Ethnograph, la presentación visual del sistema de códigos.

Organización del sistema de codificación (reubicación y agrupación de nodos)

Existe la posibilidad de agrupar varios nodos en uno nuevo 0 en uno ya existente. Esto puede efectuarse con la herramienta de búsqueda (search tool) mediante uno de los operadores $(0, y)$, el programa almacena el resultado en un nodo árbol denominado "unión", en la rama del árbol resultados de la búsqueda.

Búsqueda analítica de relaciones y pautas entre conceptos 
El trabajo con NVIVO gira alrededor de dos instrumentos principales: los exploradores y los hojeadores (browsers). Hay cuatro tipos de exploradores: de documentos, nodos, atributos y modelos. Los dos primeros proporcionan acceso sinóptico a todos los documentos y nodos, respectivamente. De modo que el investigador puede visualizarlos en conjunto, haciendo un seguimiento de estos. Dos exploradores de atributos (uno para documentos y otros para nodos) que permiten realizar funciones de creación, borrado, vista y modificación de atributos y sus valores. El explorador de modelos lista y muestra los elementos de los modelos gráficos.

\section{Documentos}

Los documentos de texto que pueden importarse al programa NVIVO son Word, pdf (este tipo de formato no se podía trabajar en versiones anteriores), texto enriquecido (rtf) y texto sin formato (txt).También se pueden importar archivos de video, multimedia, audio, imágenes y fotos digitales.

- Fases de análisis (18)

- Crear atributos y valores

- Asignar atributos y valores

- Crear categorías

- Asignar categorías

- Tablas e informes

- Modelos y diagramas

- Matrices

\section{OtROS PROGRAMAS UtILIZADOS EN LA} INVESTIGACIÓN CUALITATIVA.

Existen muchos programas para investigación cualitativa como el atlas ti, winmax, aquad, ethnograph, maxqda, entre otros. Sin embargo el programa nud*ist vivo es uno de los más completos debido a que permite realizar funciones tales como $(13,18)$ :

- funciones básicas: asignar códigos a los segmentos de textos y localizar los segmentos de textos acordes a un código y agruparlos.

- funciones de rastreo: búsqueda de códigos múltiples, es decir, segmentos a los que se les puede asignar más de un código, exploración de secuencias de códigos, es decir, segmentos que siguen a otros en algún sentido, rastreo selectivo y recuento de la frecuencia de ocurrencia o concurrencia de códigos en los datos.
- llamar archivos (recuperar documentos que han sido creados por un procesador de textos), numerar las filas de los datos textuales e imprimir copias en papel de los datos con el número de líneas asignadas.

- funciones de gestión: recuperación de archivos, salvar archivos, cambiar directorios e imprimir documentos.

En resumen permite analizar, identificar y procesar el manejo de documentos, la codificación de los datos, el manejo de datos, el modelado (representación gráfica), los informes y exportación de los datos y crear facilidades para el proyecto. Es decir este programa ofrece grandes ventajas y le ofrecen al investigador un amplio margen de uso, como por ejemplo:

- organizar y clasificar datos rápidamente

- trabajar sistemáticamente seguro de no perder ningún dato con las funciones de consulta, análisis de texto y visualización de nvivo.

- identificar conexiones sutiles de maneras que simplemente son imposibles de realizar manualmente, entre los actores o sujetos de la investigación con las categorías de interés.

- profundizar y reflexionar sobre nuevas ideas (hipótesis) en los análisis.

- soportar de manera científica y rigurosa sus conclusiones con evidencia.

- nvivo le ayuda a vincular conclusiones con los datos originales que apoyan sus ideas apoyan.

- exportar e importar datos fácilmente con aplicaciones como Microsoft Excel, Word, IBM Spss statistics y Endnote.

Es claro que el programa nud ist vivo, es uno de los más usados a nivel mundial (19) al momento de conocer percepciones, interaccionismo simbólico, fenomenológico, entre otras teorías y métodos propios de la investigación cualitativa $(20,21)$.

\section{CONCLUSIÓN}

Se conoce que la metodología tradicional de carácter positivista busca establecer las relaciones lineales de causalidad por medio de la indisoluble relación de las variables independientes y dependientes con metodologías propias de las ciencias naturales de carácter inductivo con todas sus falencias como los mencionan en sus trabajos Kuhn, Popper, Lakatos (22- 
24), lo cual lleva a construir nuevas formas de observar y por ende de investigar los fenómenos del mundo con nuevos paradigmas como el cualitativo y el de sistemas complejos, con nuevas y variadas formas de orientaciones metodológicas que privilegian de algún modo el objeto o sujeto de estudio y no el método, como suele ocurrir en el positivismo; es por esto, que en los últimos años se han utilizado en un mismo fenómeno, técnicas cualitativas para el análisis reflexivo de datos cuantitativos y viceversa y no dejarle todo a las conclusiones o resultados otorgados por los sistemas 0 software de análisis de datos considerados cualitativos o cuantitativos por muy sofisticado que se considere el software (17).

En ningún momento y por ninguna causa los software sea cual fuere debe sustituir la capacidad de inventiva de un buen análisis de investigación, el cual tampoco es excusa para un mal diseño 0 de una insuficiente 0 deficiente recolección y análisis de los datos.

Los software estadísticos 0 de análisis de datos cualitativos, están en un plano de herramientas de ayudas para las múltiples tareas para un análisis más efectivo y preciso, además de seguro, para la interpretación de una gran cantidad de datos en el menor tiempo posible y cumplir con las exigencias y parámetros establecidos por la comunidad científica y exigencias de las revistas más reconocidas a nivel mundial (17).

\section{REFERENCIAS BIBLIOGRÁFICA}

1. Millán Rodríguez F. Referencia al artículo "Uso de los análisis estadísticos en los artículos originales de Actas Urológicas Españolas: Accesibilidad para el lector.' Actas Urológicas Españolas. 2003; 27:566566.

2. Fernández Aparicio T, Miñana López B, Guzmán Martínez-Valls P, Hita Villaplana G. Uso de los análisis estadísticos en los artículos originales de Actas Urológicas Españolas: Accesibilidad para el lector. Actas U ro lógicas Españolas. 2003; 27(2):103-9.

3. Gómez OT. Una aplicación de la prueba chi cuadrado con SPSS. Industrial Data. 2008;(1):73-7.

4. Romaní F, Márquez J, Wong P. Uso de los métodos estadísticos en artículos originales de cinco revistas biomédicas peruanas. Periodo 2002-2009. Revista Peruana de Epidemiología. 2010;(2):153-60.

5. Mora-Ripoll R. Cómo mejorar la calidad estadística de los artículos presentados a revistas biomédicas: lista de comprobación para los autores. Med Clin
(Barc). 1999; 113:138-49.

6. Gavagnach M, Giménez N, Kuperstein M, Pedrazas D, Medina $E$. Formación en investigación: autopercepción de los profesionales sobre sus necesidades. Med Clin (Barc). 2009; 132(3):112-7.

7. Civil MBA, Pedagogía D, PUCE S, Lineal A. 1. Datos informativos. Teorema. 30:34.

8. Cáceres RÁ. Estadística multivariante y no paramétrica con SPSS: Aplicación a las ciencias de la salud [Internet]. Ediciones Díaz de Santos; 1994 [cited 2012 Jul 9]. Available from: http://books.qooqle.com-

Co/books?hl=es\& $\mid r=\&$ id $=$ GxhpROT-

$\mathrm{HBOC} \& 0 \mathrm{i}=\mathrm{fn} \mathrm{d} \& \mathrm{pq}=\mathrm{PR} 7 \& \mathrm{da}=\mathrm{Aplicaciones+del+SPSS+2}$ $0 . \& 0 t s=$

1U7vu74Wo\&s;q=0YDM PT3IU4VeobuWImUwwlh6Q

9. Spairani S, Alcocel EG, Roca J. Incidencia de la percepción del experto sobre las propiedades mecánicas de los materiales de construcción en el mercado inmobiliario. [cited 2012 Jul 9]; Available from: http://www-cpsv. upe, es/docum ents/COIG E2010. Pdf

10. Martín-Álvarez PJ. Prácticas de tratamiento estadístico de datos con el programa SPSS para windows: Aplicaciones en el área de Ciencia y Tecnología de Alimentos [Internet], Consejo Superior de Investigaciones Científicas; 2006 [cited 2012 Jul 9], Available from: http://books.google, com. $\mathrm{co} /$ books?hl=es\& $\mid \mathrm{r}=\& \mathrm{id}:=43$ nukPk4X2UC\&oi=fnd\&pa $=P A 11 \& d a=A p l i c$

ciones+del+SPSS+20.\&ots=kIChwR8rPJ\&sia=rPSIzWTk YlfKk5vvk c80AfK0

11. Castro MS, Aygaguer LCS. Las pruebas de significación estadística en tres revistas biomédicas: una revisión crítica. Rev Panam Salud Pública. 2004; 15:5.

12. Herreras EB. Una Herramienta Al Servicio De Perfeccionamiento Docente: NUD* 1ST VIVO. Revista de Informática Educativa y Medios Audiovisuales. 2004;1(3):21-7.

13. Herreras EB. NUD* 1ST: Vivo programa informático para el análisis de datos en investigaciones cualitativas. Pixel-Bit: Revista de medios y educación. 2005;(25):53-9.

14.Zapata-Sepúlveda $P$, López-Sánchez F, SánchezGómez MC. Content analysis research method with Nvivo-6 software in a PhD thesis: an approach to the long-term psychological effects on Chilean exprisoners survivors of experiences of torture and imprisonment. Quality \& Quantity. 2012; $1-12$. 
15. Vivara CG, Orecilla-Velillab E, Gómara-Arraizac L. “Es más difícil'": experiencias de las enfermeras sobre el cuidado del paciente con recidiva de cáncer. Enferm Clin. 2009;19(6):314-21.

16. Pagan AA. Ser (animal) humano, evolucionismo e criacionismo ñas concepgoes de alguns graduandos em Ciencias Biológicas. 2011 [cited 2012 Jul9]; Available from:

http://200.189.113.123/diaadia/diadia/arquivos/Ima ae/conteudo/artigos teses/2010/BioioaiaVte ses/ser humano.pdf

17. Teruel EGT. Soporte Informático para ja investigación cualitativa: caso de los programas Atlas, ti y NVIVO. Población y Desarrollo-Argonautas y caminantes. 2011;4(0):86-109.

18. Herreras EB. NUD* 1ST: VIVO Programa informático para el análisis de datos en investigaciones cualitativas NUD* 1ST: Lives Computer Program For The Analysis of Data in Qualitative Investigations, [cited 2013 Sep 6]; Available from: http://www.sav.us.es/pixelbit/pixelbit/articulos/n25 In25art/art2505.htm. 